\title{
An evaluation of a nurse-led rehabilitation programme (the ProBalance Programme) to improve balance and reduce fall risk of community-dwelling older people: A randomised controlled trial
}

\author{
Bruna Raquel Gouveia a,b,* , Helena Gonçalves Jardim c ${ }^{\mathrm{c}}$, Maria Manuela Martins ${ }^{\mathrm{d}}$, \\ Élvio Rúbio Gouveia ${ }^{\mathrm{e}}$, Duarte Luís de Freitas ${ }^{\mathrm{e}}$, José António Maia ${ }^{\mathrm{f}}$, \\ Debra J. Rose ${ }^{g}$

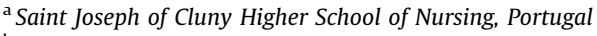 \\ ${ }^{\mathrm{b}}$ Institute of Biomedical Sciences Abel Salazar, University of Porto, Portugal \\ ${ }^{\mathrm{c}}$ Higher School of Health, University of Madeira, Portugal \\ ${ }^{\mathrm{d}}$ Higher School of Nursing of Porto, Portugal \\ ${ }^{\mathrm{e}}$ Department of Physical Education and Sports, University of Madeira, Portugal \\ ${ }_{\mathrm{f}}^{\mathrm{f}}$ CIFID, Faculty of Sport, University of Porto, Portugal \\ ${ }^{\mathrm{g}}$ Division of Kinesiology and Health Science, California State University Fullerton, USA
}

\section{A R T I C L E I N F O}

\section{Article history:}

Received 14 October 2014

Received in revised form 8 December 2015

Accepted 11 December 2015

\section{Keywords:}

Aged

Community-dwelling

Rehabilitation nursing

Postural balance

\begin{abstract}
A B S T R A C T
Objective: This study aims to assess the effect of a nurse-led rehabilitation programme (the ProBalance Programme) on balance and fall risk of community-dwelling older people from Madeira Island, Portugal.

Design: Single-blind, randomised controlled trial.

Setting: University laboratory.

Participants: Community-dwelling older people, aged 65-85, with balance impairments. Participants were randomly allocated to an intervention group (IG; $n=27$ ) or a wait-list control group (CG; $n=25$ ).

Intervention: A rehabilitation nursing programme included gait, balance, functional training, strengthening, flexibility, and 3D training. One trained rehabilitation nurse administered the group-based intervention over a period of 12 weeks ( 90 min sessions, 2 days per week). A wait-list control group was instructed to maintain their usual activities during the same time period.

Outcome: Balance was assessed using the Fullerton Advanced Balance (FAB) scale. The time points for assessment were at zero (pre-test), 12 (post-test), and 24 weeks (follow up). Results: Changes in the mean (SD) FAB scale scores immediately following the 12-week intervention were 5.15 (2.81) for the IG and -1.45 (2.80) for the CG. At follow-up, the mean (SD) change scores were -1.88 (1.84) and 0.75 (2.99) for the IG and CG, respectively. The results of a mixed between-within subjects analysis of variance, controlling for physical activity levels at baseline, revealed a significant interaction between group and time $(F(2$, $42)=27.89, p<0.001$, Partial Eta Squared $=0.57)$ and a main effect for time $(F(2$, $43)=3.76, p=0.03$, Partial Eta Squared $=0.15$ ), with both groups showing changes in the mean FAB scale scores across the three time periods. A significant main effect comparing
\end{abstract}

\footnotetext{
* Corresponding author at: Saint Joseph of Cluny Higher School of Nursing/Escola Superior de Enfermagem São José de Cluny, Rampa de Quinta de Sant' Ana n.22, 9050-535 Funchal, Madeira, Portugal. Tel.: +351 291743444/5.

E-mail address: bgouveia@esesjcluny.pt (B.R. Gouveia).
} 
the two groups $(F(1,43)=21.90, p<0.001$, Partial Eta Squared $=0.34)$ confirmed a clear positive effect of the intervention when compared to the control.

Conclusion: This study demonstrated that the rehabilitation nursing programme was effective in improving balance and reducing fall risk in a group of older people with balance impairment, immediately after the intervention. A decline in balance was observed for the IG after a period of no intervention.

Clinical Trial Registration Number: ACTRN12612000301864.

(c) 2015 Elsevier Ltd. All rights reserved.

\section{What is already known about the topic?}

- Research has shown that some types of exercise are moderately effective in improving clinical balance of older adults, immediately following an intervention.

- Conflicting evidence still exists as to the type of exercise and conditions under which interventions are effective.

\section{What this paper adds}

- This original research article describes a positive effect of a rehabilitation nursing intervention in improving the multiple dimensions of balance in older adults with balance impairments.

\section{Background}

One of the strongest modifiable risk factors associated with increased fall risk is balance impairment (Deandrea et al., 2010; Rubenstein and Josephson, 2002; Tinetti and Kumar, 2010). Impaired balance is also an important independent predictor of the transition in status from nonfaller to faller (Muir et al., 2010). Because of this association between balance impairments and increased risk of falling in older people, the effect of exercise interventions on balance, and specifically the effect of community-based group-structured exercise programmes, have been extensively studied over the past $20-25$ years (Rose, 2008). Currently, there is scientific evidence that some types of exercise, including gait, balance, coordination and functional tasks, strengthening exercise, 3D exercise and multiple exercise types, are moderately effective in improving clinical balance outcomes (Cadore et al., 2013; Howe et al., 2011). In particular, theory-driven rehabilitation programmes targeting important intrinsic risk factors associated with increased fall risk, such as balance and gait impairments and muscle weakness, have proven to be effective in reducing fall risk and improving balance among older people (Rose, 2011; Westlake and Culham, 2007).

However, there are still uncertainties related to specific characteristics of the participants targeted (i.e., balance levels at baseline), and the type, the dosage, the settings and the supervision needed during the intervention (Howe et al., 2011). Therefore, further research is needed on this topic.

From a rehabilitation nursing perspective, early identification of age-related changes and risk factors for falls, such as balance impairments, and the delivery of targeted training are essential to prevent older adults from progressing to more severe impairments or experiencing a serious fall. Therefore, the present research aims to provide evidence on the effect of a theory-driven groupbased exercise intervention (the ProBalance Programme) in a group of community-dwelling older people with balance impairments from Madeira Island, Portugal.

\section{Methods}

\subsection{Study design}

A randomised controlled trial (RCT) was conducted to assess the effect of the ProBalance Programme on balance of community-dwelling older people with balance impairment from Madeira Island, Portugal. This RCT was prospectively registered in the Australian New Zealand Clinical Trials Registry Platform and the clinical trial registration number was ACTRN12612000301864.

\subsection{Participants}

The sample consisted of 177 community-dwelling men and women aged 65-85 years old. Participants were recruited from Madeira Island, Portugal, by advertising in a regional newspaper, posters, flyers with written information, social networks, and through presentations by the main researcher in religious communities and social institutions in the city.

For participation, all volunteers were assessed for eligibility criteria in the project's laboratory in the University, by a trained group of assessors (six Registered Nurses and an expert in the field of Gerokinesiology, who coordinated the group). Key inclusion criteria were: (1) to be community-dwelling and aged 65-85 years; (2) to present balance impairments compatible with scoring 26 to 30/40 in the Fullerton Advanced Balance (FAB) scale or scoring 20 to 25/40 in the FAB scale, if not reporting falls in the past year; and (3) to be able to walk independently. Exclusion criteria included: (1) cognitive impairment (assessed by the Mini Mental State Test), and (2) any significant co-morbidities that would preclude participation, such as acute illnesses, progressive neurological diseases, stroke, and other unstable chronic conditions.

The optimal sample size calculation was based on results of previous research and the results of an earlier pilot study (4-week duration Mini-RCT), using G*Power3 (Faul et al., 2007). A priori, repeated-measures ANOVA indicated that a total sample size of 48 was needed to achieve $95 \%$ power to detect an interaction effect size of 
0.25 at the 0.05 level of significance. Although an attrition rate of zero was found in our pilot study, a potential $8 \%$ attrition rate was considered for the main RCT and a total of 52 participants were targeted in this study.

A simple randomisation process was used to allocate the eligible participants to one of the two groups, defined as the Intervention Group (IG) or the Control Group (CG). Numbers were randomly selected to form group 1 and group 2, using a random number generator software. Clusters (of two participants) were only used in the randomisation for couples and relatives, in order to prevent contamination. Blinding was used in the generation of the allocation sequence and in the assignment of the participants to one of the two groups. These tasks were carried out by an independent person.

Ethical approval of the ProBalance Project was granted by the Ethics Committee of the Health Service of the Autonomous Region of Madeira. Informed consent was provided by all participants during the enrollment phase, before any assessment. Written and verbal information about the study was given to all volunteers. All data collection and management procedures took into account the participants' right to privacy and confidentiality. The intervention was delivered to the CG after the end of the trial.

\subsection{ProBalance intervention}

ProBalance is a rehabilitation-nursing programme that includes multiple exercise types (e.g., balance, co-ordination and functional tasks training, gait training, strengthening and flexibility exercises, and 3D training).

Inspired by the FallProof Balance and Mobility Programme (Rose, 2010), the ProBalance is a theory-driven programme, adopting a multidimensional approach to balance and mobility in independent-living older people with balance impairments. It is based on a comprehensive approach the systems that contribute to balance and it targets intrinsic risk factors associated with increased fall risk, such as balance and gait impairments and muscle weakness (Rose, 2011).

The group-based intervention was administered by a rehabilitation nurse, who was responsible for delivering all exercise sessions, giving instructions on each exercise, controlling the number of repetitions and the duration of the exercise, as well as, controlling the patient's reaction to the training. The rehabilitation nurse was a registered clinical nurse specialist with expertise in the field of rehabilitation. Besides clinical experience, specific training on balance-related assessment and targeted intervention was accomplished by this nurse prior to the study. In the exercise sessions, there were other members of the intervention team (trained university students) that assisted in the exercise preparation and supervised the older people during exercise performance (1:1 ratio).

A total of 24 sessions was delivered over 12-consecutive weeks (i.e., 90-min duration, 2 days per week) between April and July 2012. This option was based on the results of previous research (Howe et al., 2011). Intensity of exercise was low to moderate and the Borg Rating Scale (Borg,
1982) was used to monitor perceived exertion. The setting was the project's laboratory at the University.

In the intervention protocol, each exercise session included six key exercise components: (1) multisensory training; addressing the visual, the somatosensory, and the vestibular system; (2) centre of gravity control training; (3) proactive and reactive postural strategy training; (4) gait pattern enhancement and variation training; (5) strengthening and endurance exercise; and (6) flexibility training. Further explanation on the rationale for this type of exercise intervention and a detailed description on the exercises have been described elsewhere (Rose, 2010). Materials such as sunglasses, aiming games (i.e., minibasketball set, velcro target with balls, soft baseball set), tape and floor marks, bean bags and parachute, light tray with plastic glasses, large print material (with familiar images), chairs, wooden benches (10 cm height), foam pads, air pads, resistance bands, weighted balls, balloons and nonweighted balls ( $10 \mathrm{~cm}$ diameter) were used in the training. In relation to pads, bands and weighted balls, progressions were done from more stable, less resistant or less heavy to less stable, more resistant or heavier materials.

Aiming to enhance adherence of the participants, two 45-min educational sessions were delivered at the beginning and in the middle of the intervention period. These sessions did not occur in the same day as the exercise sessions. This educational element addressed general aspects of fall prevention (risk factors and consequences of falls in older people and the importance of motivation and adherence to fall-prevention interventions).

The feasibility of the intervention protocol was previously verified in the pilot study.

Since the CG was a wait-list CG, participants in this group maintained their usual activities during the intervention period, and did not participate in any specific balance training (as assessed at baseline and monitored by questionnaire in each assessment).

\subsection{Outcome measures}

The primary outcome in this study was balance, assessed using the Fullerton Advanced Balance (FAB) scale, a balance assessment tool that is designed to be used when assessing the functional balance of independent older people residing in the community. Preliminary results of the psychometric evaluation of this scale's content, its convergent validity, test-retest, and intra- and inter-rater reliability, and also internal consistency were first published in 2006 (Rose et al., 2006). The content validity was based on results from a review of literature, an expert panel examination and feedback, and pilot-testing of the original scale with 15 healthy older participants. Although it was developed to assess higher functioning older people, convergent validity was obtained by comparing results with the Berg Balance Scale (BBS) assessment in 31 participants. The results of the Spearman rank correlation analysis indicated a significant $(p=0.01)$ and moderate correlation of 0.75 between the scores of both scales. The test-retest reliability coefficient for the FAB scale was 0.96 , the inter-rater reliability coefficients for the total score ranged from 0.94 to 0.97 , while the intra-rater reliability 
ranged from 0.97 to 1.00 . The homogeneity coefficients $(H)$ were 0.75 for all test items. A Rash analysis of the scale was also conducted by Klein et al. (2010) in a sample of 480 community-dwelling participants, aged 60 years and older, who were able to ambulate independently. The reliability of the scale to separate persons was 0.81 out of 1.00 and the reliability of the scale to separate items in terms of their difficulty was 0.99 out of 1.00 . A Cronbach's alpha value of 0.81 was established for the total FAB scale score, confirming its good internal consistency (Klein et al., 2010).

This is a performance-based measure that comprehensively addresses the multiple dimensions of balance, through static and dynamic balance activities performed in different sensory environments, and is considered to be useful for screening and identifying subtle changes in balance abilities, that act as precursor to an increased probability of falling (Hernandez and Rose, 2008). This balance assessment can be conducted in a relatively small area and its administration requires approximately 10$12 \mathrm{~min}$. The scale is comprised of 10 individual test items: (Item 1) standing with feet together and eyes closed; (Item 2) reaching forward to retrieve an object; (Item 3) turning in a circle; (Item 4) stepping up and over a bench; (Item 5) tandem walking; (Item 6) standing on one leg; (Item 7) standing on foam with eyes closed; (Item 8) jumping for distance; (Item 9) walking with head turns; and (Item 10) recovering from an unexpected loss of balance. Performance on each of the individual test items is scored using a 5 -point ordinal scale (0-4), with a maximum score of 40 points possible, representing an optimal balance performance. Lower scores mean lower balance performance and higher probability for falling (Hernandez and Rose, 2008; Rose et al., 2006).

The sensitivity and specificity of the FAB scale to predict the faller status (defined as persons with a history of two or more falls in the previous 12 months) has also been established by Hernandez and Rose (2008). The cut-off score of 25 out of 40 on the FAB scale produced the highest sensitivity (74.6\%) and specificity (52.6\%) in predicting the recurrent faller status, allowing to draw the conclusion that an older adult who scores 25 or lower on the FAB scale is considered to be at heightened risk for falling and in need of immediate intervention. Moreover, the probability of falling increased by $8 \%$ with each 1 -point decrease in the FAB scale.

Mean scores and mean change scores for the main outcome were analyzed in this research.

The time points for assessment were at zero (pre-test), 12 (post-test), and 24 weeks (follow up). Participants were assessed by an independent assessor who was blinded to group assignment. Prior to this RCT, the test-retest reliability in the assessment of the outcome measure was established in the pilot study (12 participants, 2 assessments within a 1-week interval). Intra-class correlation coefficient $(R)$ was 0.885 for the mean FAB scale score.

\subsection{Baseline assessments}

In this study, baseline characteristics were assessed, namely: (1) demographic characteristics (gender, age, education); (2) clinical characteristics (number of falls and number of medications); (3) cognition [Mini-Mental State Test (Folstein et al., 1975; Portuguese version by Guerreiro et al., 1994)]; (4) balance [FAB scale (Rose et al., 2006)]; (5) gait velocity, derived from the 30 -foot walk test (Rose, 2010); (6) fear of falling [Falls Efficacy Scale (Tinetti et al., 1990; Portuguese version by Melo, 2011)]; (7) functional fitness components [chair stand and arm curl, sit and reach, and 6-minute walk tests (Rikli and Jones, 2001)]; (8) physical activity level [Modified Baecke questionnaire (Voorrips et al., 1991; Portuguese version by Azevedo, 2009)]; (9) health-related quality of life (HRQL) [SF36 (Ware and Sherbourne, 1992; Portuguese version by Ribeiro, 2005)]. In addition to validating all assessment protocols in the pilot study, the test-retest reliability of all measures was also verified. Intra-class correlation coefficients $(R)$ ranged between 0.689 and 0.987 .

\subsection{Statistical analysis}

Statistical analyses included descriptive statistics and a mixed-ANOVA (between groups within subjects). This mixed between-within subjects analysis of variance was conducted to assess the impact of the intervention/control on participants' total scores on the FAB Scale, and across the three time periods (pre-test, post-test and 12-week follow-up). Physical activity at baseline was included as covariate in the model, due to its confounding part. Data analysis assumptions were verified. The level of confidence was set at 95\%. Data were analyzed using the IBM SPSS statistics 20 computer package (IBM Corp. Released, 2011).

A per protocol analysis was conducted.

\section{Results}

Advertisements started in January 2012. Recruitment started in March 2012 and was completed in April 2012. A total of 177 older adults were assessed for potential enrollment. Of these, only 52 older adults were eligible and randomly allocated to the IG $(n=27)$ or to the wait-list control group (CG), who maintained their usual activities $(n=25)$. A total of 46 participants completed the study (IG; $n=26$; CG; $n=20$ ). The intervention protocol was fully applied during the course of the study, as planned. Adherence for the participants who completed the study, assessed by the proportion of sessions attended, was $100 \%$. No adverse events (i.e., falls) or side effects were associated with the exercise intervention or the assessments. However, other events led to attrition, as described in Fig. 1.

Through a simple randomisation process, two groups with similar characteristics were formed. Participants' characteristics at baseline are summarised in Table 1 .

\subsection{Effect of the intervention}

Descriptive statistics for mean FAB Scale scores at baseline and immediately following the intervention are presented in Table 2. After 12 weeks (post-test), the change scores in the mean FAB scale were 5.15 points for the IG and -1.45 points for the CG, reaching statistical significance. Considering the predictive properties of the $\mathrm{FAB}$ 


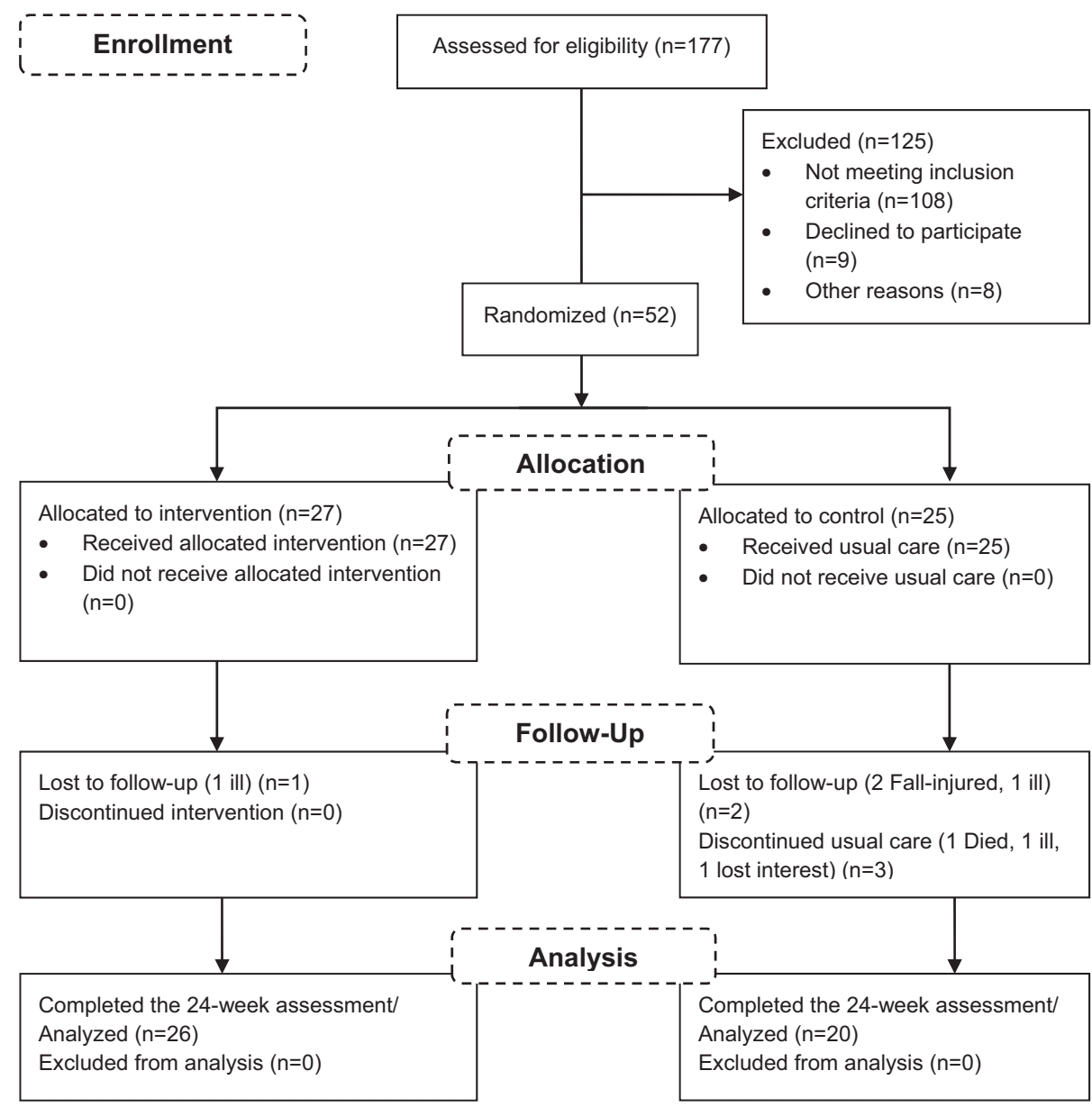

Fig. 1. Participant flow through the phases of the randomised controlled trial.

scale described by Hernandez and Rose (2008), who report that the probability of falling increased by $8 \%$ with each $1-$ point decrease in the $\mathrm{FAB}$ scale; the change score after the 12-week intervention for the CG could be associated with an increase in the probability of falling.

All participants were assessed again 12 weeks after the completion of the intervention (detraining period). Descriptive results related to the follow up are presented in Table 3. The mean FAB scale change scores were -1.88 and 0.75 points, for IG and CG, respectively. In both, CG and IG, changes reached statistical significance. Again, the mean change score in the IG could represent an increase in the probability of falling.

\subsection{Effect size estimates}

A mixed between-within subjects analysis of variance was conducted to assess the impact of the intervention/ control on participants' scores on the FAB scale, across the three time periods (pre-test, post-test, and 12-week followup), controlling for physical activity level at baseline.

This analysis revealed a significant interaction effect (Wilk's Lambda $=0.43, F(2,42)=28.89, p<0.001$, Partial Eta Squared $=0.57)$. There was also a statistically significant main effect for time (Wilk's Lambda $=0.85, F(2,42)=3.76$, $p=0.03$, Partial Eta Squared $=0.15$ ), with both groups showing changes in the mean FAB scale scores across the three time periods. However, there was a significant main effect for group $(F(1,43)=21.90, p<0.001$, Partial Eta Squared $=0.34)$, showing that the IG revealed a significant improvement in balance performance when compared to the CG. In addition, according to the guidelines proposed by Cohen (1988), the results demonstrated a very large effect size (Partial Eta Squared=0.34). The interpretation of the effects identified is supported by the graphic representation of the results for the mean FAB scale scores seen in Fig. 2.

\section{Discussion}

The general aim of this study was to examine the effect of the ProBalance programme, using a clinical measure of balance in community-dwelling older people. Two similar groups were evaluated in this RCT. The group that was randomly allocated to the 12 -week intervention showed significant gains in balance after the intervention, when compared to their peers that were randomly allocated to the CG. After the intervention (post-test), the mean FAB scale score increased by 5.15 points in the IG, while there 
Table 1

Participants' characteristics at baseline: sociodemographic, clinical, cognition, balance, functional fitness, physical activity and healthrelated quality of life.

\begin{tabular}{|c|c|c|}
\hline Variables & CG $(n=20)$ & IG $(n=26)$ \\
\hline \multicolumn{3}{|l|}{ Sociodemographic } \\
\hline Female $n$ & $22(88 \%)$ & $24(89 \%)$ \\
\hline Age in years (SD) & $74.06(4.60)$ & $73.15(4.57)$ \\
\hline Education in years (SD) & $4.05(2.52)$ & $4.15(2.29)$ \\
\hline \multicolumn{3}{|l|}{ Clinical } \\
\hline Number of medications $n(\mathrm{SD})$ & $5.50(3.02)$ & $5.81(2.55)$ \\
\hline Number of falls in the past year $n$ (SD) & $0.45(0.60)$ & $0.54(0.86)$ \\
\hline \multicolumn{3}{|l|}{ Cognition } \\
\hline Mini-Mental State score (SD) & $26.45(3.10)$ & $27.38(2.25)$ \\
\hline \multicolumn{3}{|l|}{ Balance } \\
\hline Total FAB scale score (SD) & $26.95(2.67)$ & $27.58(2.77)$ \\
\hline Gait velocity at the preferred speed in m/s (SD) & $1.16(0.25)$ & $1.28(0.17)$ \\
\hline Gait velocity at the maximum speed in $\mathrm{m} / \mathrm{s}$ (SD) & $1.53(0.34)$ & $1.67(0.21)$ \\
\hline Falls Efficacy Scale score (SD) & $86.90(18.35)$ & $90.88(10.75)$ \\
\hline \multicolumn{3}{|l|}{ Functional fitness } \\
\hline Chair stand test $n(\mathrm{SD})$ & $13.05(3.28)$ & $13.62(2.70)$ \\
\hline Arm curl test $n(\mathrm{SD})$ & $15.00(3.49)$ & $16.08(3.64)$ \\
\hline 6 -minute walk test in $\mathrm{m}(\mathrm{SD})$ & $455.36(97.72)$ & $490.62(65.73)$ \\
\hline \multicolumn{3}{|l|}{ Physical activity } \\
\hline Household score (SD) & $1.74(0.29)$ & $1.89(0.45)$ \\
\hline Sports score (SD) & $1.33(1.48)$ & $1.07(1.97)$ \\
\hline Leisure time score (SD) & $2.32(2.78)$ & $5.28(4.41)$ \\
\hline Total score (SD) & $5.38(2.50)$ & $8.24(4.92)$ \\
\hline \multicolumn{3}{|l|}{ Health-related quality of life } \\
\hline SF-36 physical component score (SD) & $190.71(56.69)$ & $220.76(62.47)$ \\
\hline SF-36 mental component score (SD) & $211.25(76.86)$ & $231.43(103.65)$ \\
\hline SF-36 total score (SD) & $401.96(115.73)$ & $452.19(151.48)$ \\
\hline
\end{tabular}

CG, control group; IG, intervention group; FAB, Fullerton Advanced Balance.

Table 2

Findings on the outcome measure at baseline and after the intervention by group: control and intervention.

\begin{tabular}{|c|c|c|c|c|c|c|c|c|}
\hline \multirow[t]{2}{*}{ Variable } & \multicolumn{4}{|c|}{ Control group $(n=20)$} & \multicolumn{4}{|c|}{ Intervention group $(n=26)$} \\
\hline & Pre-test & Post-test & $\Delta$ score & $p$ & Pre-test & Post-test & $\Delta$ score & $p$ \\
\hline $\begin{array}{l}\text { Mean FAB scale } \\
\text { score (SD) }\end{array}$ & $26.95(2.67)$ & $25.50(3.36)$ & $-1.45(2.80)$ & 0.032 & $27.58(2.77)$ & $32.73(2.11)$ & $5.15(2.81)$ & $<0.0001$ \\
\hline
\end{tabular}

FAB, Fullerton Advanced Balance (possible score range: 0-40); $\Delta$ score, change between pre- and post-test.

Table 3

Findings on the outcome measure at post-test and follow-up by group: control and intervention.

\begin{tabular}{|c|c|c|c|c|c|c|c|c|}
\hline \multirow[t]{2}{*}{ Variable } & \multicolumn{4}{|c|}{ Control group $(n=20)$} & \multicolumn{4}{|c|}{ Intervention group $(n=26)$} \\
\hline & Post-test & Follow up & $\Delta$ score & $p$ & Post-test & Follow up & $\Delta$ score & $p$ \\
\hline $\begin{array}{l}\text { Mean FAB scale } \\
\text { score (SD) }\end{array}$ & $25.50(3.36)$ & $26.25(3.89)$ & $0.75(2.99)$ & 0.276 & $32.73(2.11)$ & $30.85(3.18)$ & $-1.88(1.84)$ & $<0.0001$ \\
\hline
\end{tabular}

FAB, Fullerton Advanced Balance (possible score range: 0-40); $\Delta$ score, change between post-test and follow up.

was a decrease of 1.45 points in the CG. At follow up, the mean change scores were -1.88 and 0.75 points, for IG and CG, respectively. Although the mean FAB scale score was slightly lower for the IG at follow-up (30.85), the mean FAB score remained higher than at the baseline (27.58) (see Table 2 and Table 3). Overall, both groups showed changes in the FAB scale scores across the three time periods; nevertheless opposite results were found for the IG and CG, which is explained by the significant interaction between group and time, the significant main effect for time, and the significant main effect for group.
Similarly, Westlake and Culham (2007), in a randomised controlled trial aimed to assess the effect of an 8-week theory-driven exercise intervention based on the FallProof Programme, found post-intervention improvements in the total FAB scale score (a 4-point increase for the IG and a 1-point increase for the CG, which received education only). Contrary to our results, Westlake and Culham (2007) reported the maintenance of FAB scale scores by the participants in the IG at follow up. With regard to the increase in the total $F A B$ scale score in the CG at follow-up, a similar finding was also reported by 


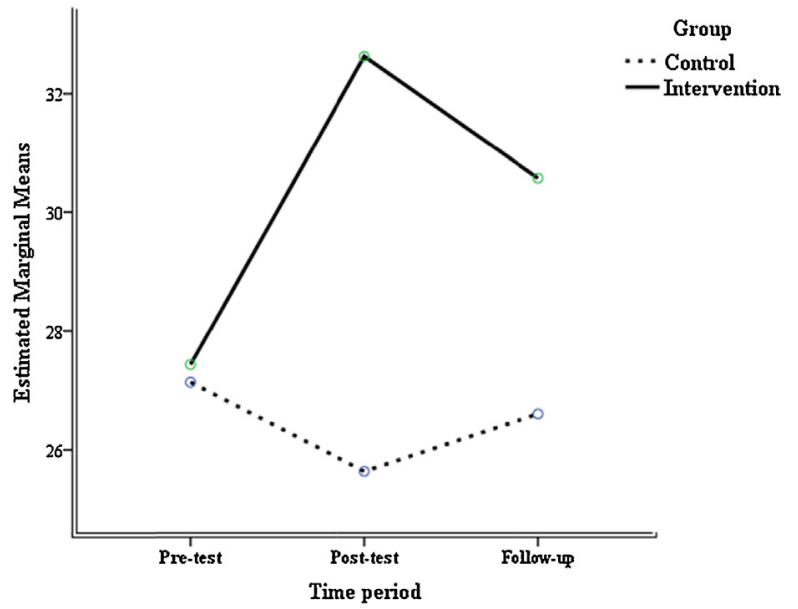

Fig. 2. Graphic representation of the results from the mixed betweenwithin subjects analysis of variance (group $\times$ time, with physical activity as covariate), showing the change in FAB scale mean scores over time for the IG and the CG.

Westlake and Culham (2007) after the intervention period. A possible explanation for these results could be the training effect after repeated assessments using the FAB scale, as well as, an increased awareness among the participants in the CG in relation to balance and fall prevention.

Although different measures of balance were used as outcome measures in the majority of randomised trials reviewed, multiple exercise type interventions have been shown to be associated with significant improvements in other composite measures of balance (Howe et al., 2011). Change score of 17.80 for the IG $(n=21)$ and 0.40 for the CG $(n=23)$ in the BBS were found by Worm et al. (2001), after a 12-week intervention. Other studies, also using the BBS as an outcome measure, reported significant improvements in mean differences between control and intervention immediately after the intervention (Beyer et al., 2007; Bieryla and Dold, 2013; Sykes and Ling, 2004). Notwithstanding using other outcome measures, other trials have also found similar findings as results of the different exercise interventions (Bateni, 2012; Kaesler et al., 2007; Nitz et al., 2010).

Another interesting finding of this study was that in the IG the mean FAB scale score increased and moved away from the cut-off point of 25 , which is predictive of the recurrent faller status. On the other hand, after 12 weeks, participants in the CG continued to obtain FAB scale scores equal to or lower than 25 points, which can be associated to a heightened risk for falling and to the need for immediate intervention (Hernandez and Rose, 2008).

The statistical significance of the effect of the intervention demonstrated responsiveness to training in the participants in the IG. A decline in balance was observed, however, for the IG following a period of no intervention, suggesting that additional or continuous intervention could be necessary to maintain or improve the balance performance of the older adults. Therefore, the current study adds evidence to support the conclusion stated by Howe et al. (2011), that positive balance effects were only evident while engaging in the intervention. Another valuable finding is that, although the FAB scale score for the IG declined at follow up, the mean FAB scale score did not return to the baseline value. These findings highlight the clinical significance of the effect of the ProBalance intervention, namely through its contribution to reducing fall risk.

\subsection{Strengths}

There is previous evidence that exercise interventions can improve balance in community-dwelling older people. Yet, most studies on this topic have targeted healthy older people and no balance screening has been performed at baseline. In addition, most trials reflected inadequate reporting of methods (Howe et al., 2011). The present report of the ProBalance randomised controlled trial, provides further evidence for the positive effects of group-based exercise interventions targeting a group of community-dwelling older people, with balance impairments identified at baseline. From a rehabilitation nursing perspective, early identification of balance impairments and the delivery of specific balance training are important preventive interventions, since many older adults only seek health professional advice when falls and serious fallrelated injuries happen. Therefore, this type of approach to balance abilities may prevent community-dwelling older people from progressing to more severe impairments or experiencing a serious fall (Yang et al., 2012).

Moreover, the present study aimed to respond to the need for research on theory-driven rehabilitation programmes that focus on manipulating individual, task, and environmental constraints concurrently, as suggested by Rose and Clark (2000), handling challenge in order to improve the individual's balance abilities when impairments in balance are identified.

One additional highlight of our study was the high adherence rate (100\%) demonstrated by the IG participants who completed the intervention. This may be indicative of the high acceptability and motivation of the participants, related to this type of intervention.

Lastly, since the present research focused on assessing balance in independent community-dwelling older people, the FAB scale was chosen as the main outcome measure, due to its high sensitivity in detecting subtle changes in balance abilities in community-dwelling older people. Besides it is a relatively new multidimensional balance assessment tool, it was designed to assess balance of higher functioning older people, has been shown to have good psychometric properties, does not require expensive equipment and is easily reproduced in community settings (Hernandez and Rose, 2008; Klein et al., 2010; Rose et al., 2006).

\subsection{Limitations}

Two limitations of this study should be addressed. First, participants were volunteers who responded to our advertisements, which may limit the generalizability of the study's findings to the wider population. Secondly, the using of $1: 1$ ratio of assistant to participation in this 
group-based exercise programme may limit its use in the clinical settings, due to the need of additional personnel.

\subsection{Overall evidence and implications}

In conclusion, this research demonstrated that the ProBalance exercise programme was effective in improving balance in a sample of older people with balance impairments, immediately after the intervention. The large effect size identified suggests that the improvements could be directly attributed to the intervention. No harm was identified. However, the programme's long-term efficacy, its effectiveness in "real world" clinical settings, and its cost-effectiveness remain to be evaluated. Future research should focus on long term-efficacy and include pragmatic trials to assess the effectiveness and cost-effectiveness of this intervention in "real world" clinical settings.

\section{Acknowledgements}

The authors would like to thank the mentioned institutions for the funding provided and also the ProBalance assessment and intervention teams for their contribution to the project. We are especially grateful to the older people who took part in this study for their participation and interest.

Conflict of interest: None declared.

Funding: Supported by ARDITI - Regional Agency for the Development of Research, Technology and Innovation (PhD Grant no. 1236/1973). Supported by the University of Madeira in the establishment of the ProBalance laboratory. Supported through the first author's involvement in the European Science Foundation Research Networking Programme 'REFLECTION' - 09-RNP-049. The views expressed are those of the authors and not necessarily those of the European Science Foundation.

Ethical approval: This trial was approved by the Madeira Regional Health Service's Ethics Committee (N.6/2011).

\section{References}

Azevedo, P.F.P., 2009. Estudo para a validação do questionário de Baecke modificado por acelerometria, na avaliação da actividade física em idosos portugueses (Master's thesis) Retrieved from U.Porto's Open Repository (12903).

Bateni, H., 2012. Changes in balance in older adults based on use of physical therapy vs the Wii Fit gaming system: a preliminary study. Physiotherapy 98 (3), 211-216.

Beyer, N., Simonsen, L., Bülow, J., et al., 2007. Old women with a recent fall history show improved muscle strength and function sustained for six months after finishing training. Aging Clin. Exp. Res. 19 (4), 300-309.

Bieryla, K.A., Dold, N.M., 2013. Feasibility of Wii Fit training to improve clinical measures of balance of balance in older adults. Clin. Interv. Aging 8, 775-781.

Borg, G., 1982. Psychophysical bases of perceived exertion. Med. Sci. Sports Exerc. 14 (5), 377-381.

Cadore, E.L., Rodríguez-Mañas, L., Sinclair, A., et al., 2013. Effects of different exercise interventions on risk of falls, gait ability, and balance in physically frail older adults: a systematic review. Rejuvenation Res. 16 (2), 105-114.

Cohen, J., 1988. Statistical Power Analysis for Behavioral Sciences, 2nd ed. Lawrence Erlbaum Associates Publishers, Hillsdale.

Deandrea, S., Lucenteforte, E., Bravi, F., et al., 2010. Risk factors for falls in community-dwelling older people: a systematic review and metaanalysis. Epidemiology 21 (5), 658-668.

Faul, F., Erdfelder, E., Lang, A.-G., et al., 2007. G*Power 3: a flexible statistical power analysis program for the social, behavioral, and biomedical sciences. Behav. Res. Methods 39, 175-191.

Folstein, M.F., Folstein, S.E., McHugh, P.R., 1975. Mini-mental state: practical method for grading the cognitive state of patients for the clinician. J. Psychiatr. Res. 12 (3), 189-198.

Guerreiro, M., Silva, A.P., Botelho, A., et al., 1994. Adaptação à população portuguesa da tradução do Mini Mental State Examination (MMSE). Rev. Port. Neurol. 1, 9

Hernandez, D., Rose, D.J., 2008. Predicting which older adults will or will not fall using the Fullerton Advanced Balance Scale. Arch. Phys. Med. Rehabil. 89, 2309-2315.

Howe, T.E., Rochester, L., Neil, F., et al., 2011. Exercise for improving balance in older people. Cochrane Database Syst. Rev. 11,, Art. No.: CD004963.

IBM Corp. Released, 2011. IBM SPSS Statistics for Windows, Version 20.0. IBM Corp., Armonk, NY.

Kaesler, D.S., Mellifont, R.B., Kelly, P.S., Taaffe, D.R., 2007. A novel balance exercise program for postural stability in older adults: a pilot study. J. Bodyw. Mov. Ther. 11 (1), 37-43.

Klein, P.J., Fiedler, R.C., Rose, D.J., 2010. Rasch analysis of the Fullerton Advanced Balance (FAB) Scale. Physiother. Can. 63 (1), 115-125.

Muir, S.W., Berg, K., Chesworth, B., et al., 2010. Modifiable risk factors identify people who transition from non-fallers to fallers in community-dwelling older adults. Physiother. Can. 62 (4), 358-367.

Nitz, J.C., Kuys, S., Isles, R., Fu, S., 2010. Is the Wii Fit a new-generation tool for improving balance, health and well-being? A pilot study. Climacteric 13 (5), 487-491.

Ribeiro, J.P., 2005. O importante é a saúde: estudo de adaptação de uma técnica de avaliação do estado de saúde-SF-36. Merck Sharp \& Dolme, Lisboa.

Rikli, R.E., Jones, J., 2001. Senior Fitness Test Manual. Development and Validation of a Functional Fitness Test for Community-Residing Older Adults. Human Kinetics, Champaign, IL.

Rose, D.J., Lucchese, N., Wiersma, L.D., 2006. Development of a multidimensional balance scale for use with functionally independent older adults. Arch. Phys. Med. Rehabil. 87, 1478-1485.

Rose, D.J., 2008. Preventing falls among older adults: no one size suits all intervention strategy. J. Rehabil. Res. Dev. 45 (8), 1153-1166.

Rose, D.J., 2010. FallProof! A Comprehensive Balance and Mobility Training Program 2nd ed. Human Kinetics, Champaign.

Rose, D.J., 2011. Reducing the risk of falls among older adults: the Fallproof Balance and Mobility Program. Curr. Sports Med. Rep. 10 (3) 151-156.

Rose, D.J., Clark, S., 2000. Can the control of bodily orientation be significantly improved in a group of older adults with a history of falls? J. Am. Geriatr. Soc. 48 (3), 275-282.

Rubenstein, L.Z., Josephson, K.R., 2002. The epidemiology of falls and syncope. Clin. Geriatr. Med. 18, 141-158.

Sykes, K., Ling, W.M., 2004. Exercise training and fall-risk prevention for community-dwelling elders. Am. J. Recreat. Ther. 3 (2), 36-42.

Tinetti, M.A., Richman, D., Powell, L., 1990. Falls efficacy as a measure of fear of falling. J. Gerontol. 45 (6), 239-243.

Tinetti, M.E., Kumar, C., 2010. The patient who falls "It's always a tradeoff". J. Am. Med. Assoc. 303 (3), 258-266.

Voorrips, L.E., Ravelli, A.C.J., Dongelmans, P.C.A., et al., 1991. A physical activity questionnaire for the elderly. Med. Sci. Sports Exerc. 23 (8), 974-979.

Ware, J.E., Sherbourne, C.D., 1992. The MOS 36-item short-form health survey (SF-36). Med. Care 30 (6), 473-483.

Westlake, K.P., Culham, E.G., 2007. Sensory-specific balance training in older adults: effect on proprioceptive reintegration and cognitive demands. Phys. Ther. 87 (10), 1274-1283.

Worm, C.H., Vad, E., Puggard, L., et al., 2001. Effects of a multicomponent exercise program on functional ability in community-dwelling, frail older adults. J. Aging Phys. Act. 9 (4), 414-424.

Yang, X.J., Hill, K., Moore, K., et al., 2012. Effectiveness of a targeted exercise intervention in reversing older people's mild balance dysfunction: a randomized controlled trial. Phys. Ther. 92 (1), 24-37. 